\title{
Differential savings, factor shares, and endogenous growth cycles
}

\author{
Volker Böhm*, Leo Kaas ${ }^{1}$
}

Department of Economics, University of Bielefeld, P.O.Box 100131, D-33501 Bielefeld, Germany

\begin{abstract}
The paper analyzes the dynamic properties of the neoclassical one-sector growth model with differential savings in the sense of Kaldor-Pasinetti. The economy exhibits unstable steady states and fluctuations if the income distribution varies sufficiently and if shareholders save more than workers. The paper analyzes in detail the dynamics for the case with a fixed proportions technology as well as with a smooth approximation. If the savings propensities differ by an arbitrarily small amount, the system exhibits topological chaos in the sense of Li and Yorke for an open set of production functions. The analytical results are supplemented by numerical experiments.
\end{abstract}

JEL classification: E25; E32; O40

Keywords: Growth; Income distribution; Business cycles

\section{Introduction}

Two versions of the standard one-sector neoclassical model of economic growth are used as basic tools to analyze fundamental issues in dynamical

\footnotetext{
* Corresponding author. This research is part of the project 'Dynamische Makroökonomik' supported by the Deutsche Forschungsgemeinschaft under contract Bo 635/8-1,2. We are indebted to two referees for useful remarks and especially to Cars Hommes for valuable and constructive criticism on earlier versions of this paper. The usual disclaimer applies.

${ }^{1}$ Present address: Institute for Advanced Studies, Department of Economics and Finance, Stumpergasse 56, A-1060 Vienna, Austria.

E-mail addresses: vboehm@wiwi.uni-bielefeld.de (V. Böhm), kaas@ihs.ac.at (L. Kaas)
} 
macroeconomics. One of them is the model of optimal growth, originated by Ramsey (1924), describing savings behavior so as to maximize the infinite discounted sum of utility of an infinitely lived representative consumer. The second one is the so-called Solow-Swan model (Swan, 1956; Solow, 1956) with a constant aggregate propensity to save out of income. Both models predict that the time paths of capital and output per capita are monotonically converging to a long run steady state under the usual neoclassical conditions (see for example Dechert, 1984). Therefore, such models preclude the possibility of cycles. As a consequence, additional economic features, such as nonconvexities in production or nonoptimal savings behavior in the Solow-Swan model, have been introduced to exhibit endogenous growth cycles. Furthermore, it is well known that any complex (chaotic) dynamic behavior can be generated within twosector models of optimal growth under an appropriate degree of discounting (Benhabib and Nishimura, 1985; Boldrin and Montrucchio, 1986).

Parallel to the early developments of the standard neoclassical growth theory, Kaldor (1956, 1957), Pasinetti (1962), Samuelson and Modigliani (1966) and others were investigating the question to what extent different savings behavior of the two income groups (labor and capital) might influence the growth path. It is obvious that different but constant savings propensities of the two income recipients make the aggregate propensity to save nonconstant and depending on the income distribution. An immediate implication from this is that the aggregate savings function need no longer be concave, so that multiple and unstable steady states can occur.

Although the question was posed originally within the Harrod-Domar model of fixed proportions (Harrod, 1939), the investigations of the literature examined primarily the role of differential simple savings behavior as determinants of long run steady states. Possible effects arising from the income distribution on stability were largely neglected. One exception is Dixit (1976) who addresses the general question of stability in both versions of the Kaldor-Pasinetti models, the neoclassical as well as the fixed coefficients model. While he argues that there may be "...equilibrium paths (which may) have strange features" (p. 61), he essentially considers the neoclassical case with smooth production as regular or stable.

All other contributions discussing instabilities and/or endogenous cycles in the one-sector model formulate aggregate savings functions using different features rather than the Kaldorian setting or income distribution effects. Day (1982) identifies a particular type of a nonlinear aggregate savings function as a source of possible chaotic dynamics. In his case neither income effects nor differential simple savings behavior is used as an economic justification. In a model with financial intermediation, Woodford (1989) shows the possibility of chaos when income distribution effects and differential savings behavior interact. His model is a boundary case of the class of models presented here. The form of the production function used combines elements of a fixed coefficients 
technology with a version reminiscent of the well-known logistic function. The role of the income distribution in that example seems transparent. However, it is not discussed.

Apart from the somewhat cursory treatment in the paper by Woodford, the role of differential simple savings behavior and distribution effects for stability of stationary states has not been investigated in the literature. This paper provides a systematic investigation of distribution effects and of simple savings behavior interacting to generate instability and topological chaos. It turns out that the necessary crucial factor is the elasticity of the marginal product of capital which exerts the relevant influence on the dynamic properties of the model. Section 2 describes the model and provides necessary conditions for the occurrence of multiple steady states and non-monotonic dynamics. Section 3 illustrates that multiple steady states and cyclical dynamics occur for Leontief-type production functions as soon as saving rates of workers and shareholders differ. In this case the mapping describing the dynamics is discontinuous. However, in spite of this fact, one finds that (almost) all cycles are stable. Section 4 considers smooth approximations of Leontief production functions. By use of the $\mathrm{Li}$ and Yorke condition it is shown that topological chaos can occur if the savings rate of shareholders exceeds that of workers slightly. A numerical bifurcation analysis investigates the role of system parameters on the dynamic behavior.

\section{The model}

Consider a standard neoclassical one-sector growth model in the tradition of Kaldor (1956,1957) and Pasinetti (1962), where the two types of agents, workers and shareholders, have possibly different but constant savings propensities. A single investment/consumption commodity is produced from labor and capital input with constant returns to scale. The production function $f: \mathbb{R}_{+} \rightarrow \mathbb{R}_{+}$, mapping capital per worker $k$ into output per worker $y$, is assumed to satisfy the weak Inada condition: ${ }^{2}$

$f$ is $C^{2}$, strictly monotonically increasing, strictly concave, and such that

$$
\lim _{k \rightarrow \infty} \frac{f(k)}{k}=0 \text { and } \quad \lim _{k \rightarrow 0} \frac{f(k)}{k}=\infty .
$$

The labor force grows at rate $n \geq 0$ and capital depreciates at rate $0<\delta \leq 1$. It is assumed that the wage rate equals the marginal product of labor which is $w(k):=f(k)-k f^{\prime}(k)$. Shareholders receive the marginal product of capital $f^{\prime}(k)$, which implies that total capital income per worker is $k f^{\prime}(k)$. Both income groups

\footnotetext{
${ }^{2}$ Notice that it is not assumed that $f(0)=0$.
} 
have a constant savings rate $0 \leq s_{\mathrm{w}} \leq 1$ for workers and $0 \leq s_{\mathrm{r}} \leq 1$ for shareholders. As a consequence, the time-one map describing capital accumulation is given by

$$
k_{t+1}=G\left(k_{t}\right):=\frac{1}{1+n}\left((1-\delta) k_{t}+s_{\mathrm{w}} w\left(k_{t}\right)+s_{\mathrm{r}} k_{t} f^{\prime}\left(k_{t}\right)\right) .
$$

Clearly, $G$ depends on $f$ as well as on the parameters $s_{\mathrm{w}}, s_{\mathrm{r}}, n, \delta$. It is obvious that the standard growth model of Solow (1956) is obtained if the two savings propensities are equal. Condition (I) then implies that there exists a unique positive steady state $\bar{k}$ and that $G$ is strictly monotonically increasing and concave. This implies that $\bar{k}$ is globally stable. As is well known, $\bar{k}$ is in general not optimal, i.e. it does not maximize long-run consumption per capita.

It is equally straightforward to see that $G$ preserves all its global properties for any $s_{\mathrm{r}} \neq s_{\mathrm{w}}$ if the income distribution, i.e. the factor shares induced by the marginal products, does not change with the capital intensity $k$. Observe that the isoelastic production function $f(k)=k^{\alpha}, 0<\alpha<1$, is the only function satisfying (I) for which the income distribution is constant. Thus, existence, uniqueness, and stability of steady states of $G$ cannot depend on differential savings, when Cobb-Douglas technologies are considered only. These issues will be analyzed in the sequel for general production functions satisfying (I) with two arbitrary savings propensities. First, the question of existence and uniqueness of steady states is investigated.

Proposition 1. (i) $k=0$ is a steady state if and only if $s_{\mathrm{w}}=0$ or $f(0)=0$.

(ii) There exists at least one positive steady state if $s_{\mathrm{r}}>0$ and $\lim _{k \rightarrow 0} f^{\prime}(k)=\infty$ or if $s_{\mathrm{w}}>0$ and $f^{\prime}(0)<\infty$.

(iii) There exists at most one positive steady state if $s_{\mathrm{r}} \geq s_{\mathrm{w}}$.

Proof. $k$ is a steady state if and only if

$$
s_{\mathrm{w}} w(k)+s_{\mathrm{r}} k f^{\prime}(k)=(n+\delta) k .
$$

(I) implies $0 \leq k f^{\prime}(k) \leq f(k)-f(0)$ and therefore $\lim _{k \rightarrow 0} k f^{\prime}(k)=0$. Thus, $k=0$ is a steady state if and only if $s_{\mathrm{w}} f(0)=0$.

On the other hand, stationarity of $\bar{k}>0$ is equivalent to

$$
s_{\mathrm{w}}\left(\frac{f(\bar{k})}{\bar{k}}-f^{\prime}(\bar{k})\right)+s_{\mathrm{r}} f^{\prime}(\bar{k})=n+\delta .
$$

(I) together with de l'Hôpital's rule yields $\lim _{k \rightarrow \infty} f^{\prime}(k)=0$. Therefore, the lefthand side tends to zero for $\bar{k} \rightarrow \infty$. For $\bar{k} \rightarrow 0$ the left-hand side tends to infinity under the assumptions made above, which gives at least one positive solution. Moreover, $s_{\mathrm{r}} \geq S_{\mathrm{w}}$ implies that the left-hand side is strictly monotonically decreasing. Hence, there cannot be more than one positive steady state. 
This proposition implies that steady states are unique if shareholders do not save less than workers. It will be shown in the next section that multiple positive steady states may occur if $s_{\mathrm{w}}>s_{\mathrm{r}}$.

Proposition 2. Assume $s_{\mathrm{w}} \geq s_{\mathrm{r}}$ or $E_{f}(k):=k f^{\prime \prime}(k) / f^{\prime}(k) \geq-1$ for all $k$. Then $G$ is monotonically increasing in $k$.

Proof. A simple computation implies that $G^{\prime}(k) \geq 0$ if and only if

$$
\left(s_{\mathrm{w}}-s_{\mathrm{r}}\right) k f^{\prime \prime}(k) \leq 1-\delta+s_{\mathrm{r}} f^{\prime}(k) .
$$

If $s_{\mathrm{w}} \geq s_{\mathrm{r}}$ this inequality is satisfied trivially. Otherwise, it follows from $k f^{\prime \prime}(k)+f^{\prime}(k) \geq 0$.

Proposition 2 implies that cyclical dynamic behavior can be excluded either if shareholders do not save more than workers or if the curvature of the production function measured by the elasticity $E_{f}$, is small in absolute value. Since

$$
\frac{\mathrm{d}\left(k f^{\prime}(k)\right)}{\mathrm{d} k}=f^{\prime}(k)\left(1+E_{f^{\prime}}(k)\right),
$$

the curvature condition of Proposition 2 is strongly related to income distribution effects: The capital income decreases with increasing capital stock, if and only if $E_{f},(k)<-1$.

While it is clear that this condition is not fulfilled for the Cobb-Douglas production function $f(k)=k^{\alpha}\left(E_{f}, \equiv \alpha-1>-1\right)$, it can occur for large classes of concave production functions. For example, if $f$ is bounded (which means that labor is not completely substitutable by capital) or if $f$ is unbounded and satisfies $\lim _{k \rightarrow \infty} f(k) / \ln (k)=0$, then $\lim _{k \rightarrow \infty} k f^{\prime}(k)=0$. Thus, capital income $k f^{\prime}(k)$ must be decreasing in $k$ for large values of $k$ in these cases.

Woodford (1989) uses an example of a bounded production function given by

$$
f(k)= \begin{cases}a k-\frac{b}{2} k^{2}+c & \text { if } k \leq \frac{a}{b}, \\ \frac{a^{2}}{2 b}+c & \text { if } k>\frac{a}{b}\end{cases}
$$

with parameters $a, b, c>0$, for which $G$ turns out to be the logistic map if $\delta=1$ and $s_{\mathrm{w}}=0$. Another example is the Leontief technology

$$
f_{\mathrm{L}}(k)=\min (a k, b)+c, \quad a, b, c>0 .
$$

Both do not satisfy (I) directly. However, they satisfy the two boundary conditions and they can be approximated by functions satisfying (I). For instance, the 
Leontief function is approximated by the family of concave production functions $^{3}$

$$
f_{\alpha}(k)=a\left(k+\alpha \ln \left(\frac{1+\mathrm{e}^{-b /(\alpha a)}}{1+\mathrm{e}^{(a k-b) /(\alpha a)}}\right)\right)+c, \quad \alpha>0 .
$$

These functions satisfy (I) for all $a, b, c, \alpha>0$. Moreover, if $k \neq b / a$ it is straightforward to show that

$$
\lim _{\alpha \rightarrow 0} f_{\alpha}(k)=f_{\mathbf{L}}(k) \quad \text { and } \quad \lim _{\alpha \rightarrow 0} f_{\alpha}^{\prime}(k)=f_{\mathbf{L}}^{\prime}(k) .
$$

Finally, the exponential function

$$
f(k)=\frac{a}{b}\left(1+c-\mathrm{e}^{-b k}\right),
$$

is another example of a bounded production function satisfying (I) for all $a, b, c>0$. It may be of independent interest to what extent differential savings and income distribution effects interact to determine the golden rule steady state. Total consumption per worker (which is proportional to consumption per capita if workers and shareholders grow at the same rate) is equal to

$$
c(k)=f(k)-s_{\mathrm{w}} w(k)-s_{\mathrm{r}} k f^{\prime}(k) .
$$

From (1), maximization of $c(k)$ over steady states with different savings rates is equivalent to the maximization of $f(k)-(n+\delta) k$ which has the necessary and sufficient first-order condition $f^{\prime}(k)=n+\delta$. Let $k^{*}=\left(f^{\prime}\right)^{-1}(n+\delta)$ denote the golden rule capital stock. Then $k^{*}$ is a steady state of the Kaldor-Pasinetti model iff

$$
s_{\mathrm{w}}\left(f\left(k^{*}\right)-(n+\delta) k^{*}\right)=(n+\delta)\left(1-s_{\mathrm{r}}\right) k^{*} .
$$

Let $E_{f}(k):=f^{\prime}(k) k / f(k)$ denote the elasticity of the production function $f$. Then, the above condition is equivalently expressed as

$$
s_{\mathrm{r}}+s_{\mathrm{w}} \frac{1-E_{f}\left(k^{*}\right)}{E_{f}\left(k^{*}\right)}=1 .
$$

Therefore, for given $n$ and $\delta$ the pairs of savings rates leading to optimal steady state consumption are described by a decreasing line crossing the point $s_{\mathrm{r}}=1, s_{\mathrm{w}}=0$. Whether optimal steady states are compatible with low values of $s_{\mathrm{r}}$ or high values of $s_{\mathrm{w}}$ depends on the elasticity of $f$ in $k^{*}$ (see Fig. 1). It is not clear, however, in which cases golden rule steady states with $s_{\mathrm{w}} \neq s_{\mathrm{r}}$ are stable.

\footnotetext{
${ }^{3}$ We are indebted to Cars Hommes for suggesting this approximation.
} 


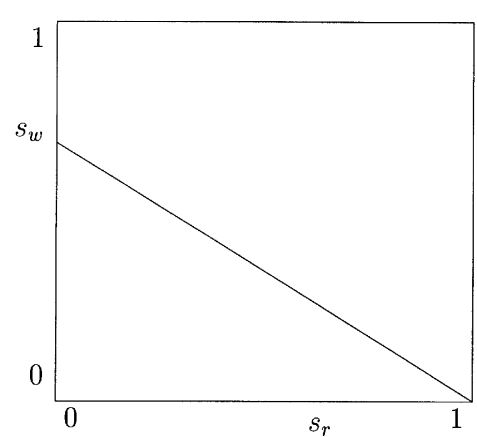

a) $E_{f}\left(k^{*}\right)<\frac{1}{2}$

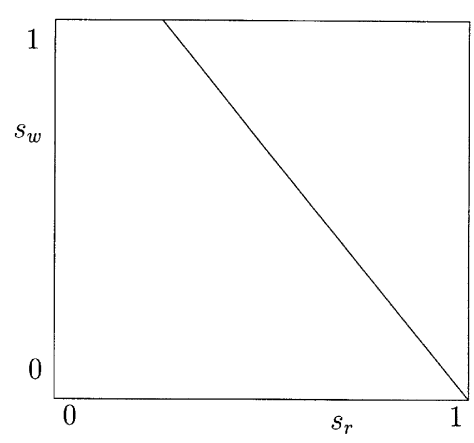

b) $E_{f}\left(k^{*}\right)>\frac{1}{2}$

Fig. 1. Savings propensities for golden rule steady state.

\section{The dynamics with fixed proportions}

This section provides a detailed analysis of the dynamic behavior for the Kaldor-Pasinetti model with a Leontief production function (3). This function is only piecewise differentiable, and the (left) derivative is $f_{\mathrm{L}}^{\prime}(k)=a$ if $k \leq b / a$ and $f^{\prime}(k)=0$ if $k>b / a$. Thus, the resulting dynamical system is described by the map

$$
G_{\mathrm{L}}(k):= \begin{cases}G_{1}(k):=\frac{1}{1+n}\left(\left(1-\delta+s_{\mathrm{r}} a\right) k+s_{\mathrm{w}} c\right), & k \leq \frac{b}{a}, \\ G_{2}(k):=\frac{1}{1+n}\left((1-\delta) k+(b+c) s_{\mathrm{w}}\right), & k>\frac{b}{a} .\end{cases}
$$

Both $G_{1}$ and $G_{2}$ are affine-linear maps with strictly positive intercept and strictly positive slope with $G_{1}^{\prime}>G_{2}^{\prime}, G_{2}^{\prime}<1$. The dynamic behavior depends crucially on the position of $G_{1}$ and $G_{2}$ at $k^{*}:=b / a$. There are four generic cases which are illustrated in Fig. 2.

It is obvious from Fig. 2 that the situations (A), (B), and (C) exhibit convergence to a unique steady state, leaving case (D) for possible cyclical behavior. Notice also that

$$
\begin{aligned}
& G_{1}\left(k^{*}\right)<G_{2}\left(k^{*}\right) \text { iff } s_{\mathrm{r}}<s_{\mathrm{w}}, \\
& G_{1}\left(k^{*}\right)<k^{*} \text { iff } s_{\mathrm{w}} c+s_{\mathrm{r}} b<(n+\delta) \frac{b}{a}, \\
& G_{2}\left(k^{*}\right)<k^{*} \text { iff } s_{\mathrm{w}}<\frac{(n+\delta) b}{a(b+c)} .
\end{aligned}
$$

Moreover, the statements of these conditions hold equally if the inequality signs " $<$ " are replaced by " $>$ ". With this observation it turns out that case (A) in 


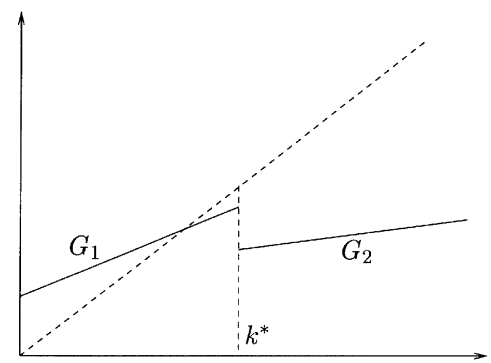

(A) Unique stable steady state (low capital)

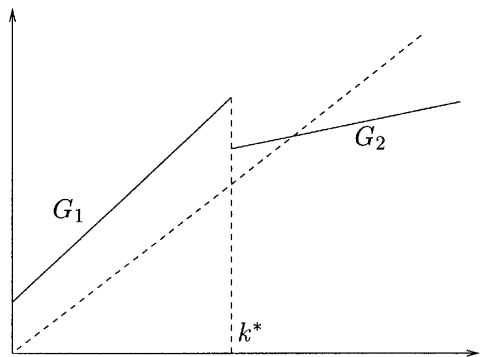

(C) Unique stable steady state (high capital)

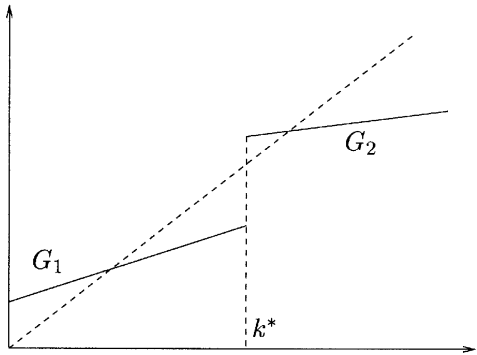

(B) Two stable steady states

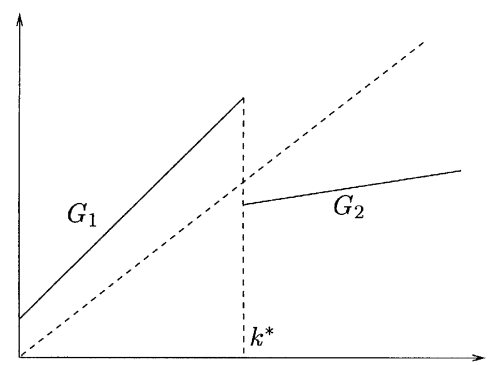

(D) No stable steady state

Fig. 2. Types of time-one maps with Leontief technology.

Fig. 2 prevails if (8) and (9) are fulfilled, that (C) prevails if (8) and (9) are fulfilled with " $>$ ", and that (B) and (D) prevail if one of (8) and (9) holds with " $<$ ", and one with " $>$ ". Given the parameters $n, \delta, a, b$ and $c$, the classification of these cases can then be illustrated in the $\left(s_{\mathrm{r}}, s_{\mathrm{w}}\right)$-diagram of Fig. 3 .

It is intuitively obvious that one obtains a similar characterization of these four generic situations if the Leontief production function is approximated by smooth functions, e.g. by the one given in (4). Let $G_{\alpha}$ denote the time-one map for the approximation of the Leontief production function $f_{\alpha}$. Because of (5), it follows $G_{\alpha}(k) \rightarrow G_{\mathrm{L}}(k)$ for all $k \neq k^{*}$ if $\alpha \rightarrow 0$. Thus, in case of either (B) or (D) in Fig. 2 either two stable steady states or no stable steady state exist for the map $G_{\alpha}$ with $\alpha$ sufficiently small. Notice further that given $s_{\mathrm{w}}>s_{\mathrm{r}}$ there exists a value of the technology parameter $a$ such that $\left(s_{\mathrm{r}}, s_{\mathrm{w}}\right)$ falls into region (B) of Fig. 3. In addition, for $s_{\mathrm{w}}<s_{\mathrm{r}}$ there exists a value of $a$ such that $\left(s_{\mathrm{r}}, s_{\mathrm{w}}\right)$ falls into region (D). From these observations one obtains

Proposition 3. Let $n \geq 0$ and $0<\delta<1$. If $1 \geq s_{\mathrm{w}}>s_{\mathrm{r}} \geq 0$, there exists a production function satisfying (I) such that $G$ has multiple steady states. If $0 \leq s_{\mathrm{w}}<s_{\mathrm{r}} \leq 1$, there exists a production function satisfying (I) such that $G$ has no stable steady state. 


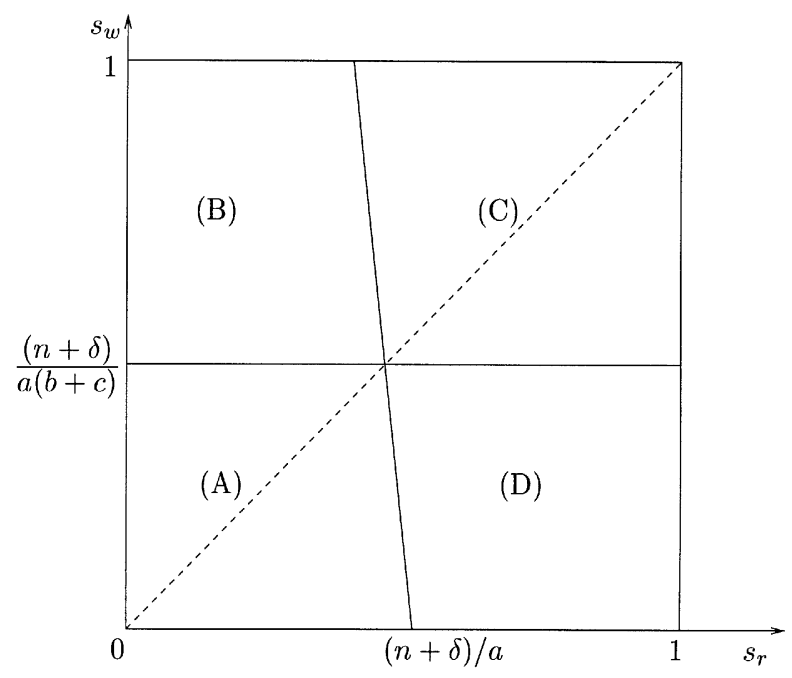

Fig. 3. Stability regions for the Leontief technology.

While each trajectory in cases (A), (B) and (C) converges to a steady state, case (D) exhibits more interesting dynamic behavior. For the Leontief technology with the discontinuous map $G_{\mathrm{L}}$ each trajectory converges to the invariant, attracting and compact interval $\left[G_{2}\left(k^{*}\right), G_{1}\left(k^{*}\right)\right]$. If this interval contains some cycle which does not hit $k^{*}$, this cycle must be globally stable, as the following proposition shows:

Proposition 4. Let $K_{\tau}=\left(k_{s}\right)_{s=1, \ldots, \tau}$ be a cycle of order $\tau$ for $G_{\mathrm{L}}$ such that $k_{s} \neq k^{*}$ for all $s=1, \ldots, \tau$. Then $K_{\tau}$ is globally stable.

Proof. It can be shown easily that $G_{1}\left(G_{2}\left(k^{*}\right)\right)>G_{2}\left(G_{1}\left(k^{*}\right)\right)$ in case (D). Thus, $G_{\mathrm{L}}$ is injective on the interval $\left[G_{2}\left(k^{*}\right), G_{1}\left(k^{*}\right)\right]$. Observe that a discontinuity of the $\tau$ th iterate $G_{\mathrm{L}}^{\tau}$ in $\left[G_{2}\left(k^{*}\right), G_{1}\left(k^{*}\right)\right]$ must be either $k^{*}$ or a preimage of $k^{*}$ under some $G_{\mathrm{L}}^{s}, s=1, \ldots, \tau-1$. Since each $G_{\mathrm{L}}^{s}$ is injective there are at most $\tau$ discontinuities of $G_{\mathrm{L}}^{\tau}$ in $\left[G_{2}\left(k^{*}\right), G_{1}\left(k^{*}\right)\right]$. This implies that there is a partition of $\left[G_{2}\left(k^{*}\right), G_{1}\left(k^{*}\right)\right]$ into intervals $I_{s}, s=1, \ldots, m$ with $m \leq \tau+1$, such that $G_{\mathrm{L}}^{\tau}$ is affine-linear on each $I_{s}$. Since the two affine-linear maps $G_{1}$ and $G_{2}$ defining $G_{\mathrm{L}}$ are of the form $G_{i}(k)=\alpha_{i}+\beta_{i} k$ with $\alpha_{i}>0, \beta_{i}>0, i=1,2, G_{\mathrm{L}}^{\tau}$ can be written $G_{\mathrm{L}}^{\tau}(k)=A_{s}+B_{s} k$ for $k \in I_{s}$ with positive constants $A_{s}$ and $B_{s}$. Now, if $\left(k_{s}\right)_{s=1, \ldots, \tau}$ is a cycle of order $\tau$, each $k_{s}$ must be contained in the interior of one of the above intervals (implying $m \geq \tau$ ). Assume w.l.o.g. that $k_{s} \in I_{s}, s=1, \ldots, \tau$. From $k_{s}=G_{\mathrm{L}}^{\tau}\left(k_{s}\right)=A_{s}+B_{s} k_{s}$ and $A_{s}, k_{s}>0$ it follows $B_{s}<1$. This implies that each trajectory starting in $\bigcup_{s=1}^{\tau} I_{s}$ converges to $K_{\tau}$ and that $K_{\tau}$ is locally 


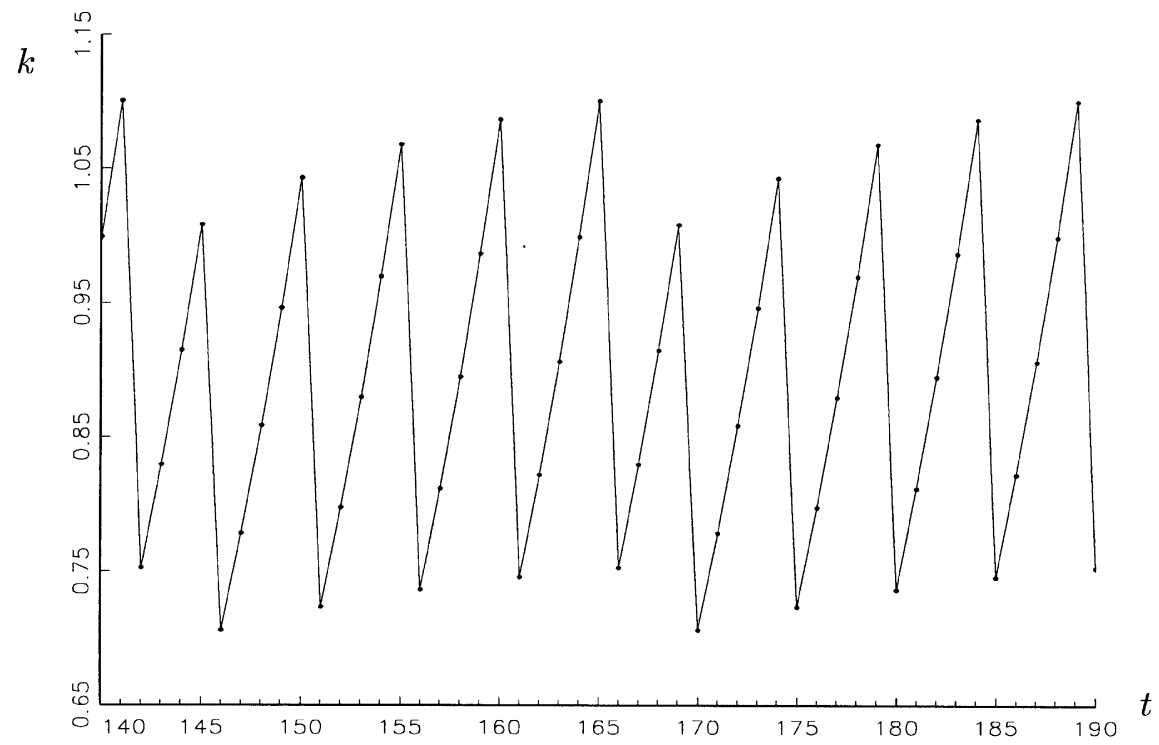

Fig. 4. A cycle of period 24 for the Leontief technology and $a=b=1, c=0.01, n=0, \delta=0.5$, $s_{\mathrm{r}}=0.6$ and $s_{\mathrm{w}}=0.2$.

asymptotically stable. If $m=\tau+1, G_{\mathrm{L}}^{\tau}$ cannot have a fixpoint in $I_{m}$ (otherwise there would exist another cycle of order $\tau^{\prime}>1$ with $\tau^{\prime} \mid \tau$ which is impossible). Hence, $G_{\mathrm{L}}^{\tau}(k)>k$ or $G_{\mathrm{L}}^{\tau}(k)<k$ for all $k \in I_{m}$, and each trajectory enters eventually $\cup_{s=1}^{\tau} I_{s}$. Therefore, $K_{\tau}$ is a globally stable cycle.

This proposition states the surprising fact that almost all cycles of $G_{\mathrm{L}}$ must be globally stable. Cycles which hit $k^{*}$ cannot be locally stable, but, according to the above proof, they are still globally attractive. Keener (1980) investigates the dynamics of a class of piecewise continuous one-dimensional maps to which our map $G_{\mathrm{L}}$ belongs. ${ }^{4}$ The injectivity of the map $G_{\mathrm{L}}$ on the interval $\left[G_{2}\left(k^{*}\right), G_{1}\left(k^{*}\right)\right]$ corresponds to the nonoverlapping case considered by Keener. He shows that in this case the dynamics is periodic, except for parameter values in a Cantor set of measure zero. For the exceptional parameters, the dynamics cannot be chaotic either, but it converges to a Cantor set. We did not observe such a dynamics in our simulations. However, we did find many stable cycles of different and very high order (see also Fig. 6(a)) with some interesting patterns of regularity. Some alternate between the intervals $k<k^{*}$ and $k>k^{*}$ only once, while others alternate between them several times, spending an (almost) equal number of periods in each interval between the switches. As an example, Fig. 4 displays the

\footnotetext{
${ }^{4}$ We thank Cars Hommes for providing this reference.
} 
time series of a cycle of period 24 which switches 5 times from the interval $k<k^{*}=1$ to the interval $k>k^{*}$. There are 4 "subcycles" of order 5 and one of order 4 . Along these subcycles, the capital stock rises slowly over 4 or 3 periods within the interval $k<k^{*}=1$ until it exceeds $k^{*}$. In this situation, the marginal product of capital drops to zero which leads, because of $s_{\mathrm{r}}>s_{\mathrm{w}}$, to a sudden decline of savings, pushing the capital stock down and below $k^{*}$ again.

\section{Chaos and bifurcations}

The stability result of Proposition 4 obtained for the Leontief technology cannot be extended to smooth approximations of this production function. In particular, there may exist unstable cycles since the derivative of $G_{\alpha}$ along the cycle may become very large in absolute value if the cycle is close to $k^{*}$. A smooth (or even continuous) approximation of $G_{\mathrm{L}}$ allows the application of known results to prove the existence of chaos and infinitely periodic orbits.

Consider again the general situation of $s_{\mathrm{r}}>S_{\mathrm{w}}$ with a unique unstable positive steady state $\bar{k}$ and a non-monotonic continuous time-one map $G$. Since $G(k)>k$ for $k<\bar{k}$ and $G(k)<k$ for $k>\bar{k}$, the interval $\left[G\left(k_{\min }\right), G\left(k_{\max }\right)\right]$ with

$$
k_{\min }:=\operatorname{argmin}\{G(k) \mid k \geq \bar{k}\}
$$

and

$$
k_{\max }:=\operatorname{argmax}\{G(k) \mid k \in[0, \bar{k}]\}
$$

is a compact, invariant and attracting set. Therefore, there exists a compact attractor $A$, i.e. a minimal invariant and attracting set. Since there is only a single positive steady state which is locally unstable, $A$ consists of at least two elements. Hence, the global dynamics may be periodic or chaotic.

To show the existence of topological chaos, we make use of the well-known theorem of $\mathrm{Li}$ and Yorke (1975). If $G$ is continuous and if there exists some $k_{0}$ such that

$$
G\left(k_{0}\right)<k_{0}<G^{3}\left(k_{0}\right)<G^{2}\left(k_{0}\right),
$$

then a cycle of period 3 exists, which implies the existence of topological chaos according to the definition of $\mathrm{Li}$ and Yorke.

Proposition 5. For all $n \geq 0,0<\delta<1, s_{\mathrm{r}}>0$ and $s_{\mathrm{w}}=s_{\mathrm{r}}-\varepsilon \geq 0$ with $\varepsilon>0$, there exists an open set (in the $C^{1}$ topology) of production functions $f$ satisfying (I) such that $G$ exhibits topological chaos.

Proof. Consider first the Leontief production function $f_{\mathrm{L}}$ defined by (3) for which the time-one map is (7). Given $b$ and $c$, for each $\gamma>0$ there is an $a=a(\gamma)$ 


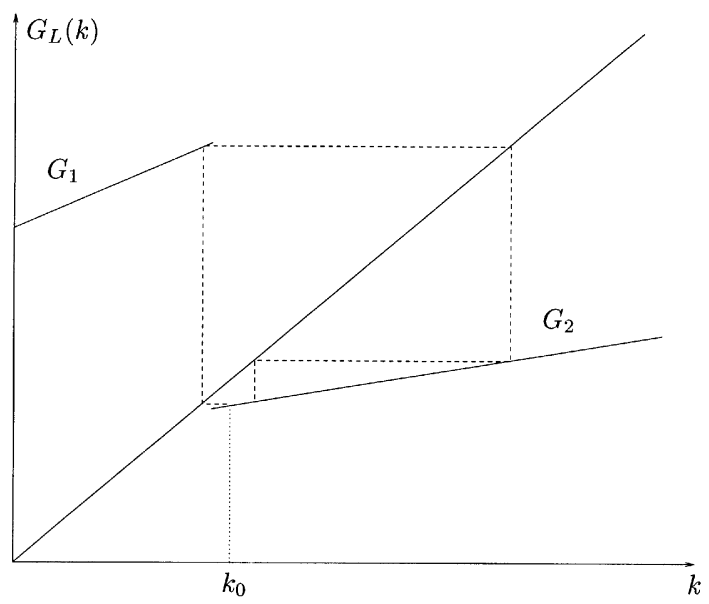

Fig. 5. Li and Yorke condition for the Leontief technology.

such that $G_{2}(b / a)=b / a-\gamma$, in particular one has $a(\gamma):=(n+\delta) b((1+n) \gamma+$ $\left.\left(s_{\mathrm{r}}-\varepsilon\right)(b+c)\right)^{-1}$. Furthermore, $G_{1}(b / a)=G_{2}(b / a)+\varepsilon b /(1+n)$. Therefore, for $\gamma$ sufficiently small, one has $G_{2}(b / a)<b / a<G_{1}(b / a)$ with $b / a-G_{2}(b / a)$ small and $G_{1}(b / a)-b / a$ close to $\varepsilon b /(1+n)$. In particular, by choosing $\gamma$ sufficiently small and $k_{0}>b / a$ close to $b / a$ one has (see Fig. 5)

$$
G_{\mathrm{L}}\left(k_{0}\right)<b / a<k_{0}<G_{\mathrm{L}}^{3}\left(k_{0}\right)<G_{\mathrm{L}}^{2}\left(k_{0}\right) .
$$

Second, because of (5) it follows for $\alpha$ sufficiently small that

$$
G_{\alpha}\left(k_{0}\right)<k_{0}<G_{\alpha}^{3}\left(k_{0}\right)<G_{\alpha}^{2}\left(k_{0}\right) .
$$

Furthermore, for $f$ sufficiently close (in a $C^{1}$ sense) to $f_{\alpha}$, the induced time-one map $G$ satisfies (10).

Proposition 5 implies that complex dynamics can occur as soon as workers have a slightly lower savings rate than shareholders. The occurrence of period3 -cycles is not an exceptional feature of production functions close to the Leontief function. Cycles of period 3 exist clearly for the production function used by Woodford (1989) and for the exponential technology (6). In the latter case the $\mathrm{Li}$ and Yorke condition can be verified for $a=400, b=1, c=0.01$, $s_{\mathrm{w}}=0, s_{\mathrm{r}}=1, n=0.07$ and $\delta=0.5$ with $k_{0}=8.72968$. It turns out that in this case a higher excess of the shareholders' savings rate over the workers' savings rate is needed for complex dynamics.

The theorem of Li and Yorke implies the existence of periodic orbits of arbitrary periods as well as chaotic trajectories. However, the theorem says 
nothing about the observability of chaotic or specific periodic trajectories if the system starts at arbitrary initial conditions. Therefore, the dynamic behavior for different parameter values and initial conditions has to be analyzed numerically. All subsequent numerical calculations were carried out using MACRODYN, a package for the simulation of discrete time dynamical systems developed by Böhm et al. (1997).

The first set of experiments with the production functions (3) and (4) showed the same long run characteristics for different initial conditions. Even though we did not find evidence of coexisting stable cycles for these technologies, they are generally not impossible in this model. ${ }^{5}$

The investigation centered primarily on the influence of the two savings propensities on the long run dynamic behavior. Fig. 6 contains two cycle cartograms, displaying the results of a two-parameter bifurcation qualitatively. The color assignment identifies the long run characteristics of the dynamical system for the given point in the parameter space.

Fig. 6(a) shows a large diversity of cycles of different orders for the Leontief technology, system parameters $n=0, \delta=0.1, a=0.2, b=1, c=0.01$ and initial value $k_{0}=5$ for varying $s_{\mathrm{w}}$ and $s_{\mathrm{r}}$. For these values, the intersection of the curves defining the stability regions in Fig. 3 is at $s_{\mathrm{w}}=s_{\mathrm{r}}=0.495$. Therefore, the range of the two parameters chosen for Fig. 6 coincides (almost) exactly with region (D). The yellow color indicates that no cyclical behavior is found, which is due to long transient behavior. Fig. 6(b) shows the equivalent cartogram for the approximation of the Leontief production function with $\alpha=0.01$ and the same parameter set and initial value. In this case, the transition from stable steady states to cyclical or chaotic dynamics occurs via period doubling cascades. The yellow color indicates again that no cycle has been found, but in most cases this corresponds to a chaotic attractor.

Fig. 6(b) indicates also that there exist several period doubling as well as period halving cascades. This is a typical phenomenon of one-dimensional dynamical systems generated by bimodal maps for which other economic applications have been given (e.g. Hommes, 1994; Prskawetz and Feichtinger, 1995). The emergence of two period doubling and two period halving cascades occurs in Fig. 6 if both savings rates increase from the lower left to the upper right corner, i.e. if a transition from case (A) to case (C) via case (D) in Fig. 2 takes place. The same bifurcation phenomenon occurs also for changes of the depreciation rate. An example is shown in Fig. 7 for the same parameter set and initial value as above, but with $s_{\mathrm{r}}=0.8$ and $s_{\mathrm{w}}=0.4$.

\footnotetext{
${ }^{5} \mathrm{We}$ are grateful to a referee for having pointed out the emergence of coexisting stable cycles of period 4 and 8 for the production function (6) and parameters $a=330, b=1, c=0.01$, $n=0.07, d=0.95, s_{\mathrm{w}}=0, s_{\mathrm{r}}=1$.
} 


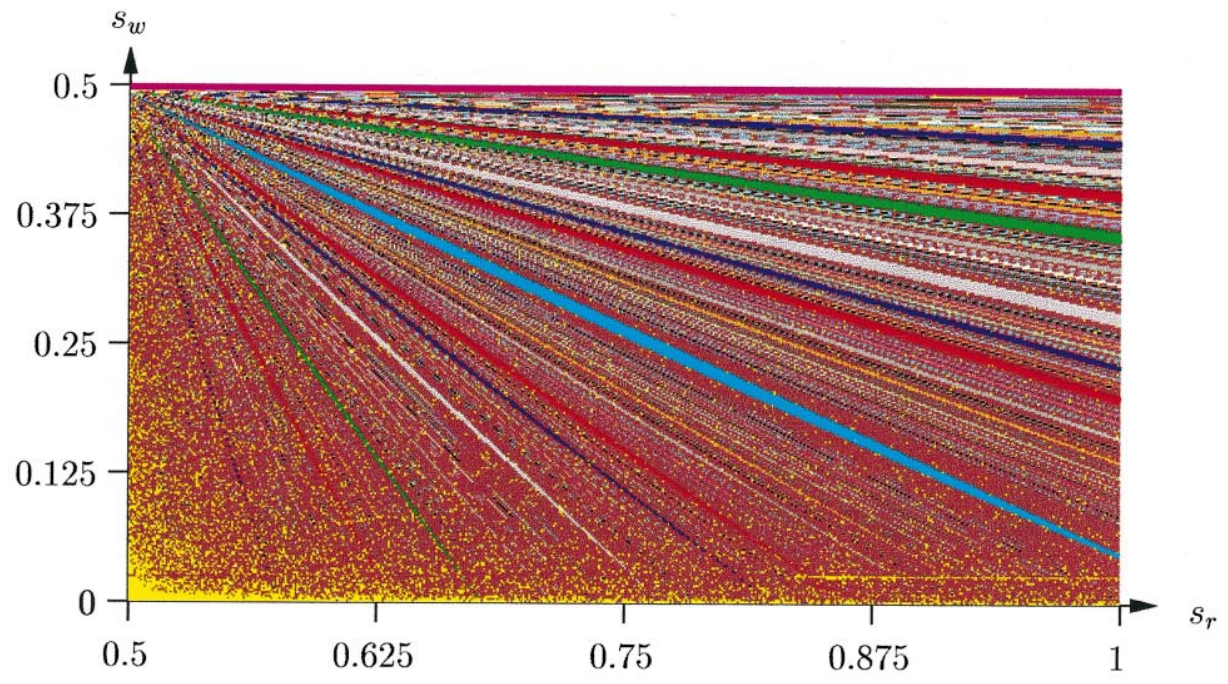

(a) Leontief technology

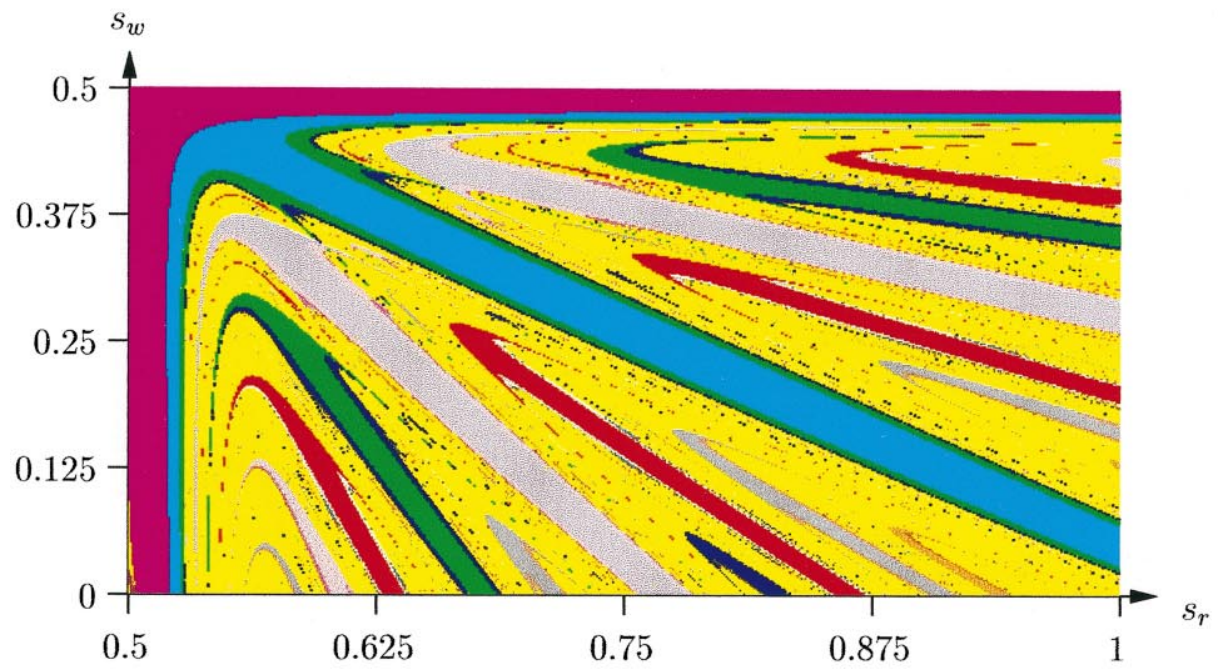

(b) Approximation with $\alpha=0.01$

\begin{tabular}{|c|c|c|c|c|c|c|c|}
\hline Order 1 & $r 2$ & Order 3 & ar 4 & ar 5 & Order 6 & Order 7 & Or \\
\hline Order 9 & Order 10 & Orde & & & & & \\
\hline Order 17 & Order 18 & Order 19 & Order 20 & Order 21 & & Order 23 & 24 \\
\hline Order 25 & Order 26 & Order 27 & Order 28 & Order 29 & Order 30 & oor & No $p$ \\
\hline
\end{tabular}

Fig. 6. Cycle cartograms in $\left(s_{\mathrm{r}}, s_{\mathrm{w}}\right)$ for $a=0.2, b=1, c=0.01, n=0, \delta=0.1$ and $k_{0}=5$. 


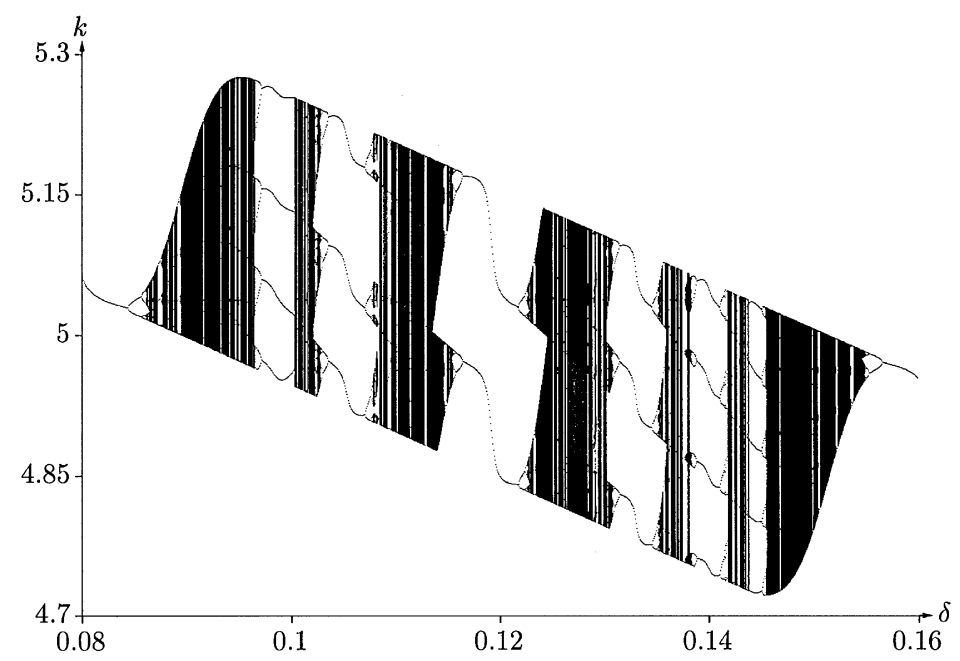

Fig. 7. Bifurcation diagram in $\delta$ for $a=0.2, b=1, c=0.01, n=0, s_{\mathrm{r}}=0.8, s_{\mathrm{w}}=0.4$ and $k_{0}=5$.

\section{Summary and conclusions}

The results of this paper show that even the simplest one-sector model of economic growth can display cyclical and chaotic dynamic behavior, if distributional effects of competitive factor pricing are coupled with constant differential savings propensities. As our analysis shows, this is neither a degenerate phenomenon in the space of parameters nor in the space of smooth production functions satisfying the weak Inada condition. Since the oscillations in this model are derived from simple savings behavior and features of the technology, it does not seem to be too far fetched to conjecture that many other neoclassical growth models might have such a dynamic behavior, once distributional effects are integrated. Thus, varying income distributions may be one of the primary causes of endogenously generated business cycles.

\section{References}

Benhabib, J., Nishimura, K., 1985. Competitive equilibrium cycles. Journal of Economic Theory 35, 284-306.

Böhm, V., Lohmann, M., Middelberg, U., 1997. MACRODYN — a dynamical systems toolkit. Department of Economics, University of Bielefeld.

Boldrin, M., Montrucchio, L., 1986. On the indeterminacy of capital accumulation paths. Journal of Economic Theory 40, 26-39.

Day, R.H., 1982. Irregular growth cycles. American Economic Review 72, 406-414. 
Dechert, W.D., 1984. Does optimal growth preclude chaos? A theorem on monotonicity. Zeitschrift für Nationalökonomie 44, 57-61.

Dixit, A., 1976. The Theory of Equilibrium Growth. Oxford University Press, London.

Harrod, R.F., 1939. An essay in dynamic theory. Economic Journal 49, 14-33.

Hommes, C., 1994. Dynamics of the cobweb model with adaptive expectations and nonlinear supply and demand. Journal of Economic Behavior and Organization 24, 315-335.

Kaldor, N., 1956. Alternative theories of distribution. Review of Economic Studies 23, 83-100.

Kaldor, N., 1957. A model of economic growth. Economic Journal 67, 591-624.

Keener, J.P., 1980. Chaotic behavior in piecewise continuous difference equations. Transactions of the American Mathematical Society 261, 589-604.

Li, T.-Y., Yorke, J.A., 1975. Period three implies chaos. American Mathematical Monthly 82, 985-992.

Pasinetti, L.L., 1962. Rate of profit and income distribution in relation to the rate of economic growth. Review of Economic Studies 29, 267-279.

Prskawetz, A., Feichtinger, G., 1995. Endogenous population growth may imply chaos. Journal of Population Economics 8, 59-80.

Samuelson, P.A., Modigliani, F., 1966. The Pasinetti paradox in neoclassical and more general models. Review of Economic Studies 33, 269-301.

Solow, R.M., 1956. A contribution to the theory of economic growth. Quarterly Journal of Economics 70, 65-94.

Swan, T.W., 1956. Economic growth and capital accumulation. Economic Record 32, 334-361.

Woodford, M., 1989. Imperfect financial intermediation and complex dynamics. In: Barnett et al. (Eds.), Economic Complexity: Chaos, Sunspots, Bubbles, and Nonlinearity. Cambridge University Press, Cambridge. 